\title{
Alternating-Offer Bargaining with Endogenous Commitment
}

\author{
Zhixian Yu
}

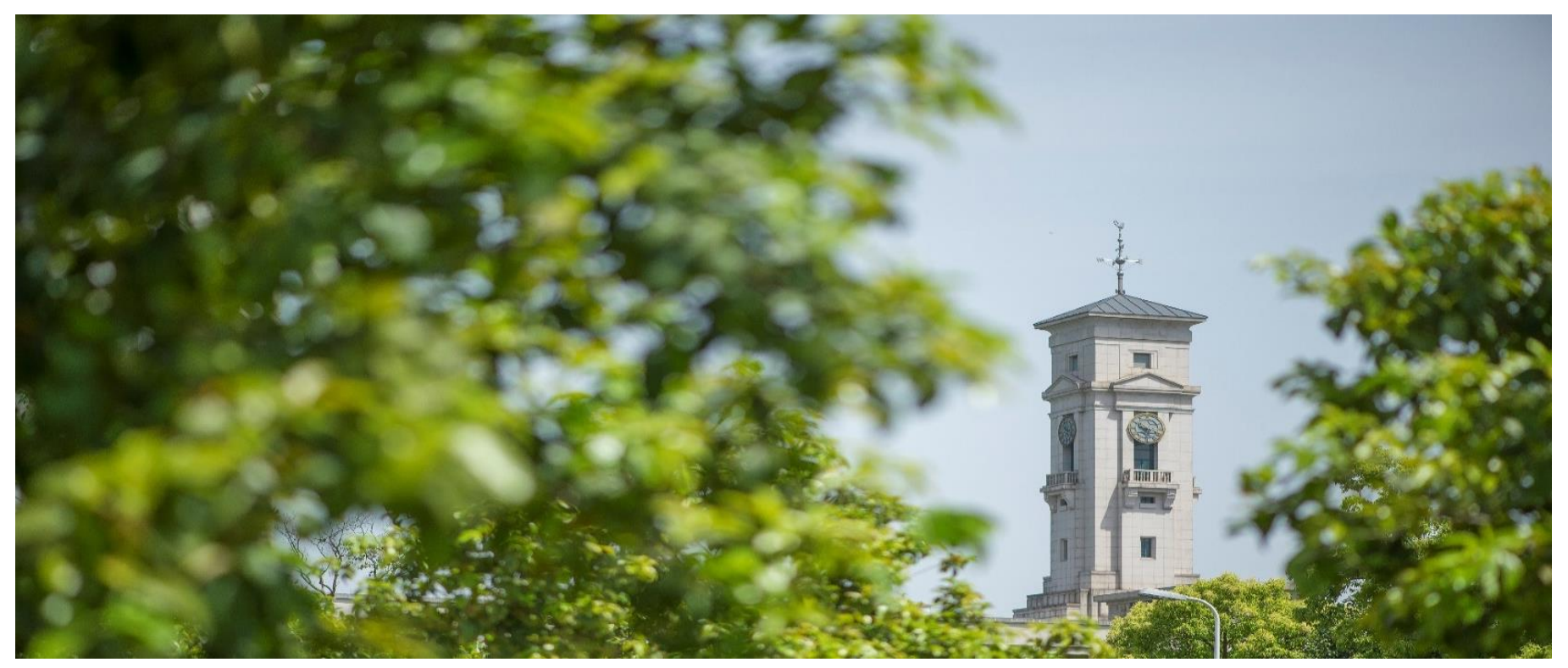


Faculty of Business, University of Nottingham Ningbo China, 199 Taikang East Road, Ningbo, 315100, Zhejiang, China.

First published 2020

This work is made available under the terms of the Creative Commons Attribution 4.0 International License:

http://creativecommons.org/licenses/by/4.0

The work is licenced to the University of Nottingham Ningbo China under the Global University Publication Licence:

https://www.nottingham.edu.cn/en/library/documents/researchsupport/global-university-publications-licence-2.0.pdf 


\title{
Alternating-Offer Bargaining with Endogenous Commitment
}

\author{
Zhixian $\mathrm{Yu}^{*}$ \\ University of Nottingham Ningbo China, Business School, 199 Taikang East Road, \\ Ningbo, 315100, China
}

\begin{abstract}
We revisit the classical alternating-offer bargaining model, further assuming that players cannot reduce their proposals during the game. In equilibrium, players have history-dependent strategies and do not necessarily reach an agreement immediately in the first stage.
\end{abstract}

JEL classification: C78

Keywords: Bargaining, Endogenous commitment, alternating-offer bargaining game, History-dependence

\section{Introduction}

The commitment whereby once an individual has made an offer they cannot later make worse offers is proposed by Fershtman and Seidmann (1993). They term this endogenous commitment, and apply it in a finite horizon, randomizing-offer bargaining model to explain the deadline effect in negotiation: Players don't reach an agreement until the deadline when the discount factor is large enough. In this paper, we adopt their assumption and apply it in an alternating-offer environment. We also find a (possible) delay in the agreement, but only for one stage.

Endogenous commitment has been discussed in various papers and interpreted from different perspectives. Fershtman and Seidmann (1993) mainly focus on the negotiator's prestige: Negotiators suffer from a reputation loss when they accept worse offers after rejecting better ones. Schwartz and Wen (2007) value good faith in bargaining: Given the signal a worse offer sends, an arbitrator may even refuse to take it to the other side. Compte and Jehiel (2003) and

\footnotetext{
*The author wishes to thank Chen Qu, Kang Rong and Jack Fanning, the editor and the anonymous referee for their helpful recommendations. Special thanks to his Ph.D. supervisor, Daniel Seidmann and Silvia Sonderegger. Telephone: +86(0)57488188630. Email address: Zhixian.Yu@nottingham.edu.cn
} 
Li (2007) use reference point theory to support their endogenous commitmentlike assumptions: Anything below the best offer they have rejected would be valued negatively. ${ }^{1}$

\section{Model}

The basic structure of our model is as follows. Two players $i=1,2$ split $\$ 1 .^{2}$ The set of all feasible agreements is $Z=\{(z, 1-z): z \in[0,1]\}$, in which $z$ refers to 1's share of the $\$ 1$ and $1-z$ refers to 2 's. The game continues for at most $T<+\infty$ stages, at which point the negotiation ends with both players earning 0 payoff. The players have a common discount factor $\delta \in(0,1)$, so each player's payoff is $U_{1}=\delta^{t} z$ and $U_{2}=\delta^{t}(1-z)$, respectively, if an agreement is reached by stage $t \in[1,2,3 \ldots T]$. The two players alternate in making proposals: 1 offers $x_{t} \in[0,1]$ to 2 (the agreement is $\left(1-x_{t}, x_{t}\right)$ if $x_{t}$ is accepted) or 2 offers $y_{t} \in[0,1]$ to 1 (the agreement is $\left(y_{t}, 1-y_{t}\right)$ if $y_{t}$ is accepted).

Until now, we have defined a classical finite horizon alternating-offer bargaining model $\Gamma$ as in Stahl (1972). We now formally define endogenous commitment in this paper ${ }^{3}$ :

Assumption 1. A proposer is not allowed to propose a share that is lower than their previous proposal, i.e., $\forall t \in[3, T], x_{t} \in\left[x_{t-2}, 1\right]$ and $y_{t} \in\left[y_{t-2}, 1\right]$.

With Assumption 1 applied in $\Gamma$, we have an extended bargaining model $\Gamma^{\prime}{ }^{4}$ We will analyze $\Gamma^{\prime}$ by characterizing its subgame perfect equilibrium, or "equilibrium" for short. Without loss of generality, we suppose that 2 is the final proposer in $\Gamma$ and $\Gamma^{\prime}$, so 1 (2) is the first proposer if $T$ is even (odd).

\section{Equilibrium}

In $\Gamma$, in equilibrium, 1 offers $x_{t}=\delta \frac{1-(-\delta)^{T-t}}{1+\delta}$ and accepts if and only if $y_{t} \geq$ $\delta \frac{1-(-\delta)^{T-t}}{1+\delta} .2$ 's equilibrium strategy is similar. It's easy to see that 2's proposals

\footnotetext{
${ }^{1}$ Compte and Jehiel (2003) study a bargaining protocol with different phases. The updating of the reference point happens only when the game enters a new phase, which occurs with a probability after each rejection. Li (2007) takes players' discounted values of the rejected offers - rather than the rejected offers - to be the reference points.

${ }^{2}$ For convenience, we refer to 1 as male and 2 as female.

${ }^{3}$ For comparison, Fershtman and Seidmann (1993) define endogenous commitment from the respondent's perspective. However, the two definitions are equivalent.

${ }^{4}$ If we consider $T=+\infty$ instead, we will be extending Rubinstein's (1982) infinite horizon alternating-offer bargaining model. In this case, in equilibrium, 1 offers $x_{t}=$ $\max \left(x_{t-2}, \min \left(\frac{\delta}{1+\delta}, \delta\left(1-y_{t-1}\right)\right)\right)$ and accepts if and only if $y_{t} \geq \min \left(\frac{\delta}{1+\delta}, \delta\left(1-x_{t-1}\right)\right)$. 2 's equilibrium strategy is similar. The equilibrium strategies differ slightly from those in Rubinstein's model, while the equilibrium outcome is the same. This extended model coincides with (1) the subscription game in Admati and Perry (1991), when the total cost $K$ is equal to each individual player's revenue $V$; $(2)$ the bargaining game in Driesen et al. (2012), when the coefficient of risk aversion goes to infinity; and (3) the wage-negotiation game in Schwartz and Wen (2007), when the outside option is $\frac{\delta}{1+\delta}$.
} 
decrease with time - hence violate endogenous commitment - so it is conceivable that $\Gamma$ and $\Gamma^{\prime}$ have quite different equilibria.

Before we discuss the equilibrium of $\Gamma^{\prime}$, we will define several terms. Let $y_{t}^{\#}=\min \left(\frac{\delta}{1+\delta}, \delta\left(1-x_{t-1}\right)\right)$ denote 1's minimal acceptable offers. Let $x_{t}^{\#}=$ $\min \left(\frac{\delta}{1+\delta}, \delta\left(1-y_{t-1}\right)\right)$ and $x_{t}^{\# \#}=\min \left(\max \left(\delta^{T-t}\left(1-y_{t-1}\right), \frac{\delta}{1+\delta}\right), \delta\left(1-y_{t-1}\right)\right)$ denote 2's minimal acceptable offers under different conditions. Let $\delta_{t}^{*}$ denote the threshold discount factors when $t \leq T-2$. $\delta_{t}^{*}$ solves $(1+\delta) \delta^{T-t-1}=1$ when $T-t$ is odd, and $(1+\delta) \delta^{T-t}=1$ when $T-t$ is even. ${ }^{5}$ Finally, let $y_{t-2}^{*}=\min \left(1-\frac{1}{(1+\delta) \delta^{T-t}}, 1-\frac{1-\delta\left(1-x_{t-1}\right)}{\delta^{T-t}}\right)$ denote 2's threshold previous offers, indicating that 2 chooses different strategies in $t$ when her proposal in $t-2$ goes across $y_{t-2}^{*}$.

Let $(f, g)$ be a pair of strategies for 1 and 2 , respectively. The following is a detailed description of $(f, g)$.

$$
\begin{aligned}
& f: \quad \text { player } 1 \\
& t \leq T-2 \quad \text { offers } x_{t}=\max \left(x_{t-2}, x_{t}^{\#}\right) \text { when } \delta \leq \delta_{t}^{*} \text {, } \\
& \text { offers } x_{t}=\max \left(x_{t-2}, x_{t}^{\# \#}\right) \text { when } \delta>\delta_{t}^{*} \text {, } \\
& \text { accepts if and only if } y_{t} \geq y_{t}^{\#} \text {. } \\
& t=T-1 \quad \text { offers } x_{T-1}=\max \left(x_{T-3}, \delta\left(1-y_{T-2}\right)\right) \text {. } \\
& t=T \quad \text { accepts if and only if } y_{T} \geq 0 \text {. } \\
& g: \quad \text { player } 2 \\
& t \leq T-2 \quad \text { offers } y_{t}=\max \left(y_{t-2}, y_{t}^{\#}\right) \text { when } \delta \leq \delta_{t}^{*} \text {, or when } \delta>\delta_{t}^{*} \text { and } y_{t-2} \geq y_{t-2}^{*} \text {, } \\
& \text { offers } y_{t}=y_{t-2} \text { when } \delta>\delta_{t}^{*} \text { and } y_{t-2}<y_{t-2}^{*} \text {, } \\
& \text { accepts if and only if } x_{t} \geq x_{t}^{\#} \text { when } \delta \leq \delta_{t}^{*} \text {, } \\
& \text { accepts if and only if } x_{t} \geq x_{t}^{\# \#} \text { when } \delta>\delta_{t}^{*} \text {. } \\
& t=T-1 \quad \text { accepts if and only if } x_{T-1} \geq \delta\left(1-y_{T-2}\right) \text {. } \\
& t=T \quad \text { offers } y_{T}=y_{T-2} .
\end{aligned}
$$

See Figure 1 for a better demonstration of $(f, g)$. The horizontal line represents the value of $\delta$; above the line is the proposer's equilibrium strategy and below is the respondent's. Notice that $\delta_{t-1}^{*}>\delta_{t}^{*}=\delta_{t+1}^{*}>\delta_{t+2}^{*}$.

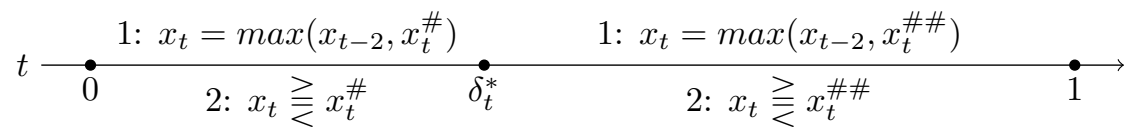

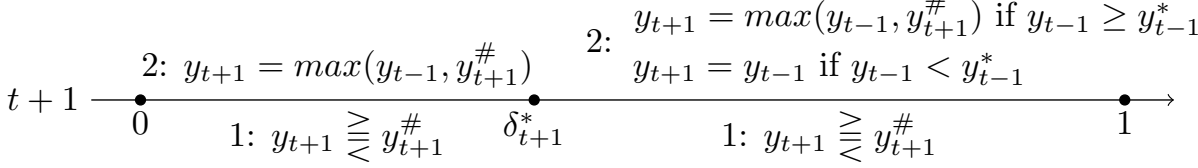

Figure 1: Equilibrium strategies in two typical consecutive stages

Suppose we have the following tie-breaking rule: A proposer makes the ac-

\footnotetext{
${ }^{5}$ Note that $\delta_{t}^{*}$ decreases with time every two stages. For example, $\delta_{t}^{T-5}=\delta_{t}^{T-4}>\delta_{t}^{T-3}=$ $\delta_{t}^{T-2}$.
} 
ceptable offer whenever he (she) is indifferent between making an acceptable offer or an unacceptable offer, and a respondent accepts whenever he (she) is indifferent between accepting or rejecting an offer. Then we have following proposition. ${ }^{6}$

Proposition 1. $(f, g)$ is the unique SPE of $\Gamma^{\prime}$. In equilibrium, the agreement is reached in the first or second stage of the game.

As the equilibrium strategies of $\Gamma^{\prime},(f, g)$ has following properties:

1. When $\delta \leq \delta^{*},(f, g)$ coincides with the equilibrium strategies in the infinite horizon bargaining (see footnote 4). Intuitively, the deadline doesn't take effect when it is relatively far away.

2. When $\delta>\delta^{*}$, the deadline kicks in. $x_{t}^{\# \#}$, which is weakly greater, replaces $x_{t}^{\#}$ when 1 proposes. Intuitively, if no agreement has been reached, an agreement of $\left(y_{T-2}, 1-y_{T-2}\right)$ will be reached in $T$; hence 2 won't accept anything less than $\delta^{T-t}\left(1-y_{t-1}\right)$ in $t$.

3. (Continue with case 2) Anticipating that, 2 may deliberately make an unacceptable offer when she proposes and wait for 1's counteroffer in the next stage. She is less likely to do so in stage $t$ if either $x_{t-1}$ or $y_{t-2}$ is higher, as shown in the condition $y_{t-2} \gtreqless y_{t-2}^{*}$ in $g$. Intuitively, here 2 is comparing her payoffs $1-y_{t}^{\#}$ (from making an acceptable offer) and $\delta^{T-t}\left(1-y_{t-2}\right)$ (from making an unacceptable offer). ${ }^{7} \mathrm{~A}$ higher $x_{t-1}$ means that 1 will accept a lower $y_{t}^{\#}$, and a higher $y_{t-2}$ means that 2 earns less from delaying the agreement.

From $(f, g)$, we can derive the equilibrium paths. When $\delta \leq \delta_{1}^{*}$, the first proposer offers $\frac{\delta}{1+\delta}$ to the opponent in the first stage. When $\delta>\delta_{1}^{*}, 1$ makes the first acceptable offer which makes 2 indifferent between accepting and waiting to take the whole surplus at $T$ : If $T$ is even, 1 offers $\delta^{T-1}$ to 2 in the first stage; if $T$ is odd, 2 offers nothing (and gets rejected, obviously) in the first stage, then accepts the counteroffer of $\delta^{T-2}$ in the second stage. See Table 1 as a summary.

\begin{tabular}{|c|c|c|}
\hline & $T$ is odd & $T$ is even \\
\hline$\delta \leq \delta_{1}^{*}$ & $\left(\frac{\delta}{1+\delta}, \frac{1}{1+\delta}\right)$ & $\left(\frac{1}{1+\delta}, \frac{\delta}{1+\delta}\right)$ \\
\hline$\delta>\delta_{1}^{*}$ & $\left(\delta-\delta^{T-1}, \delta^{T-1}\right)$ & $\left(1-\delta^{T-1}, \delta^{T-1}\right)$ \\
\hline
\end{tabular}

Table 1: Equilibrium payoffs under different conditions

\footnotetext{
${ }^{6}$ The tie-breaking rule simplifies but doesn't change the main result generically. We provide a detailed proof of Proposition 1 in Appendix A and discuss the equilibrium without the tiebreaking rule at the end of the proof.

${ }^{7}$ See case $(\mathrm{K})$ of the proof in Appendix A for details.
} 


\section{Concluding Remarks}

Through the introduction of endogenous commitment, our model captures a simple fact in bargaining: negotiation history matters. A bargainer faces the following trade-off when making proposals: A higher offer is more likely to be accepted, but it also limits one's choices in the future and weakens one's bargaining power if it gets rejected. Our model, together with Fershtman and Seidmann's (1993), explains the delay in bargaining when information is complete and is applicable to the environments in which the bargaining history is easily traced and used.

The difference between our paper and Fershtman and Sedimann's is as follows. In their model, each player has an equal chance to propose/respond in each stage of the game, meaning that both players might benefit from being the final proposer. They have equal incentives to delay to the deadline, and they do so when the discount factor is large enough. In our paper, only one player benefits from being the last proposer. Thus delay may happen, but only for one stage.

In our model, both first- and last-proposer advantage exist, see Figure 2 for a demonstration. Notice that 2 is always the final proposer, and is also the first proposer only in the right graph. Clearly, when $\delta \leq \delta_{1}^{*}$, there is first-proposer advantage, as in Rubinstein (1982). When $\delta>\delta_{1}^{*}$, the last-proposer advantage starts to overwhelm (but doesn't eliminate) the first-proposer advantage.
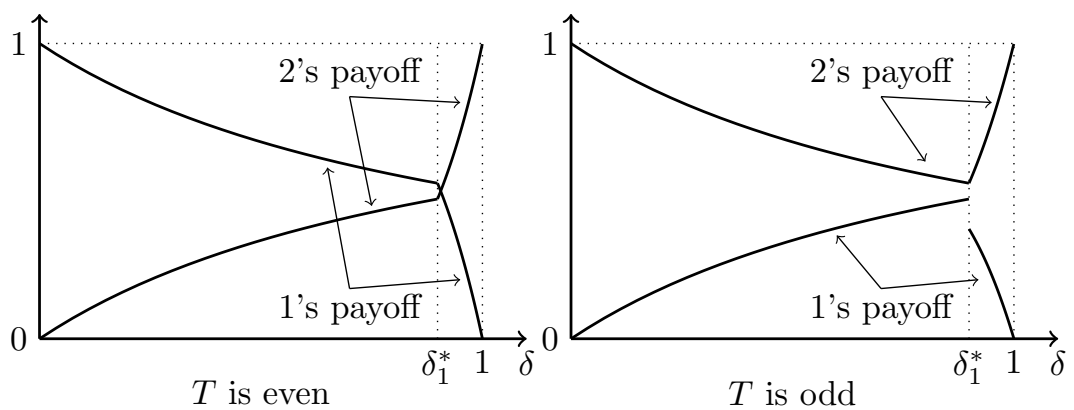

Figure 2: Equilibrium payoffs as function of the discount factor

\section{A Proof of Proposition 1}

Proof. We use backward induction to show that $(f, g)$ constitutes a sub-game perfect equilibrium. Given the tie-breaking rule, the uniqueness of equilibrium is an immediate application of Zermelo's theorem.

In $T, 1$ accepts any offer $y_{T} \geq 0$, so 2 makes the minimal offer she is bound to make, which is $y_{T}=y_{T-2}$.

In $T-1,2$ accepts if and only if $x_{T-1} \geq \delta\left(1-y_{T-2}\right)$. If $x_{T-3} \geq \delta\left(1-y_{T-2}\right)$, then 1 can only make an acceptable offer $x_{T-1}=x_{T-3}$. If $x_{T-3}<\delta\left(1-y_{T-2}\right)$, 
then he can either make an acceptable or an unacceptable offer. When 1 offers $x_{T-1} \geq \delta\left(1-y_{T-2}\right)$, the offer is accepted immediately and he earns a payoff of $1-x_{T-1}$; otherwise, the offer is rejected and he accepts 2 's counteroffer in $T$, earning a payoff of $\delta y_{T-2}$. Therefore, 1 offers $x_{T-1}=\delta\left(1-y_{T-2}\right)$ if and only if $1-\delta\left(1-y_{T-2}\right) \geq \delta y_{T-2}$, which is clearly always valid. To sum up, 1 offers $x_{T-1}=\max \left(x_{T-3}, \delta\left(1-y_{T-2}\right)\right)$.

In $T-2,1$ accepts if and only if $y_{T-2} \geq \delta\left(1-\max \left(x_{T-3}, \delta\left(1-y_{T-2}\right)\right)\right)$, namely, $y_{T-2} \geq y_{T-2}^{\#}$. If $y_{T-4} \geq y_{T-2}^{\#}$, then 2 can only make an acceptable offer $y_{T-2}=y_{T-4}$. If $y_{T-4}<y_{T-2}^{\#}$, then she can either make an acceptable or an unacceptable offer. When 2 offers $y_{T-2} \geq y_{T-2}^{\#}$, the offer is accepted immediately and she earns a payoff of $1-y_{T-2}$; otherwise, the offer is rejected and she accepts 1's counteroffer in $T-1$, earning a payoff of $\delta \max \left(x_{t-3}, \delta\left(1-y_{T-2}\right)\right)$. Therefore, 2 offers $y_{T-2}=y_{T-2}^{\#}$ if $1-y_{T-2}^{\#} \geq \delta \max \left(x_{T-3}, \delta\left(1-y_{T-4}\right)\right)$ and $y_{T-2}=y_{T-4}$ otherwise. $^{8} 1-y_{T-2}^{\#} \geq \delta \max \left(x_{T-3}, \delta\left(1-y_{T-4}\right)\right)$ is equivalent to $y_{T-4} \geq y_{T-4}^{*}=\min \left(1-\frac{1}{(1+\delta) \delta^{2}}, 1-\frac{1-\delta\left(1-x_{T-3}\right)}{\delta^{2}}\right)$. Notice that (a) when $\delta \leq \delta_{T-2}^{*}, \delta^{2}+\delta^{3} \leq 1$, so $y_{T-4}^{*} \leq 0$ and the inequality must be valid; and (b) when $\delta>\delta_{T-2}^{*}$ and $y_{T-4}<y_{T-4}^{*}, y_{T-4}<1-\frac{1-\delta\left(1-x_{T-3}\right)}{\delta^{2}}$. Hence $x_{T-3}<1-\frac{1-\delta^{2}\left(1-y_{T-4}\right)}{\delta}<\delta\left(1-y_{T-4}\right)$, so 2 's payoff when making an unacceptable offer becomes $\delta^{2}\left(1-y_{T-4}\right)$ and she offers $y_{T-2}=y_{T-4}$. To sum up, 2 offers $y_{T-2}=\max \left(y_{T-4}, y_{T-2}^{\#}\right)$ when $\delta \leq \delta_{T-2}^{*}$, or when $\delta>\delta_{T-2}^{*}$ and $y_{T-4} \geq y_{T-4}^{*}$; she offers $y_{T-2}=y_{T-4}$ otherwise.

Now we show that $(f, g)$ specifies best responses in $t$ if it does so in every $t^{\prime}>t$.

Suppose that 1 proposes in $t$. In $t^{\prime}>t$ : When $\delta \leq \delta_{t+1}^{*}$, or when $\delta>\delta_{t+1}^{*}$ and $y_{t-1} \geq y_{t-1}^{*}, 1$ accepts $y_{t+1}=y_{t+1}^{\#}$ in $t+1$ and the bargaining outcome is $\left(\max \left(y_{t-1}, y_{t+1}^{\#}\right), 1-\max \left(y_{t-1}, y_{t+1}^{\#}\right)\right)$. When $\delta>\delta_{t+1}^{*}$ and $y_{t-1}<y_{t-1}^{*}, 2$ accepts $x_{t+2}=x_{t+2}^{\# \#}$ in $t+2\left(1\right.$ rejects $y_{t+1}=y_{t-1}$ in $\left.t+1\right)$ and the bargaining outcome is $\left(1-\max \left(x_{t}, x_{t+2}^{\# \#}\right), \max \left(x_{t}, x_{t+2}^{\# \#}\right)\right)$. We first study 2 's best responses in $t$.

(A) When $\delta \leq \delta_{t}^{*}, 2$ accepts if and only if $x_{t} \geq \delta\left(1-\max \left(y_{t-1}, y_{t+1}^{\#}\right)\right)$; namely, $x_{t} \geq x_{t}^{\#}=\min \left(\frac{\delta}{1+\delta}, \delta\left(1-y_{t-1}\right)\right)$.

(B) When $\delta>\delta_{t}^{*}$, if 1 offers $x_{t}$ such that $y_{t-1} \geq y_{t-1}^{*}$, then 2 accepts if and only if $x_{t} \geq \delta\left(1-\max \left(y_{t-1}, y_{t+1}^{\#}\right)\right)$; namely, $x_{t} \geq x_{t}^{\#}$. Combining with $y_{t-1} \geq y_{t-1}^{*}, 2$ accepts if (a) $x_{t} \geq 1-\frac{1-\delta^{T-t-1}\left(1-y_{t-1}\right)}{\delta}$ and $y_{t-1} \leq 1-\frac{1}{(1+\delta) \delta^{T-t-1}}$, or (b) $x_{t} \geq \frac{\delta}{1+\delta}$ and $1-\frac{1}{(1+\delta) \delta^{T-t-1}}<y_{t-1} \leq \frac{\delta}{1+\delta}$, or (c) $x_{t} \geq \delta\left(1-y_{t-1}\right)$ and $y_{t-1}>\frac{\delta}{1+\delta}$. 2 rejects if (a) $x_{t}<\frac{\delta}{1+\delta}$ and $1-\frac{1}{(1+\delta) \delta^{T-t-1}} \leq y_{t-1} \leq \frac{\delta}{1+\delta}$, or (b) $x_{t}<\delta\left(1-y_{t-1}\right)$ and $y_{t-1}>\frac{\delta}{1+\delta}$.

\footnotetext{
${ }^{8}$ Notice that 2's payoff when making an unacceptable offer, $\delta \max \left(x_{t-3}, \delta\left(1-y_{T-2}\right)\right)$, is weakly decreasing in $y_{T-2} .2$ earns weakly lower payoffs when he offers $y_{T-2}>y_{T-4}$. This applies to all similar cases in the following analysis, i.e., inequality 1 to inequality 6 .
} 
(C) When $\delta>\delta_{t}^{*}$, if 1 offers $x_{t}$ such that $y_{t-1}<y_{t-1}^{*}$, then 2 accepts if and only if $x_{t} \geq \delta^{2} \max \left(x_{t}, x_{t+2}^{\# \#}\right)$; namely, $x_{t} \geq \delta^{2} \min \left(\max \left(\delta^{T-t-2}(1-\right.\right.$ $\left.\left.\left.\left.y_{t-1}\right), \frac{\delta}{1+\delta}\right), \delta\left(1-y_{t-1}\right)\right)\right)$. Combining with $y_{t-1}<y_{t-1}^{*}, 2$ accepts if $\delta^{T-t}(1-$ $\left.y_{t-1}\right) \leq x_{t}<1-\frac{1-\delta^{T-t-1}\left(1-y_{t-1}\right)}{\delta}$ and $y_{t-1}<1-\frac{1}{(1+\delta) \delta^{T-t-1}}$. 2 rejects if $x_{t}<\delta^{T-t}\left(1-y_{t-1}\right)$ and $y_{t-1}<1-\frac{1}{(1+\delta) \delta^{T-t-1}}$.

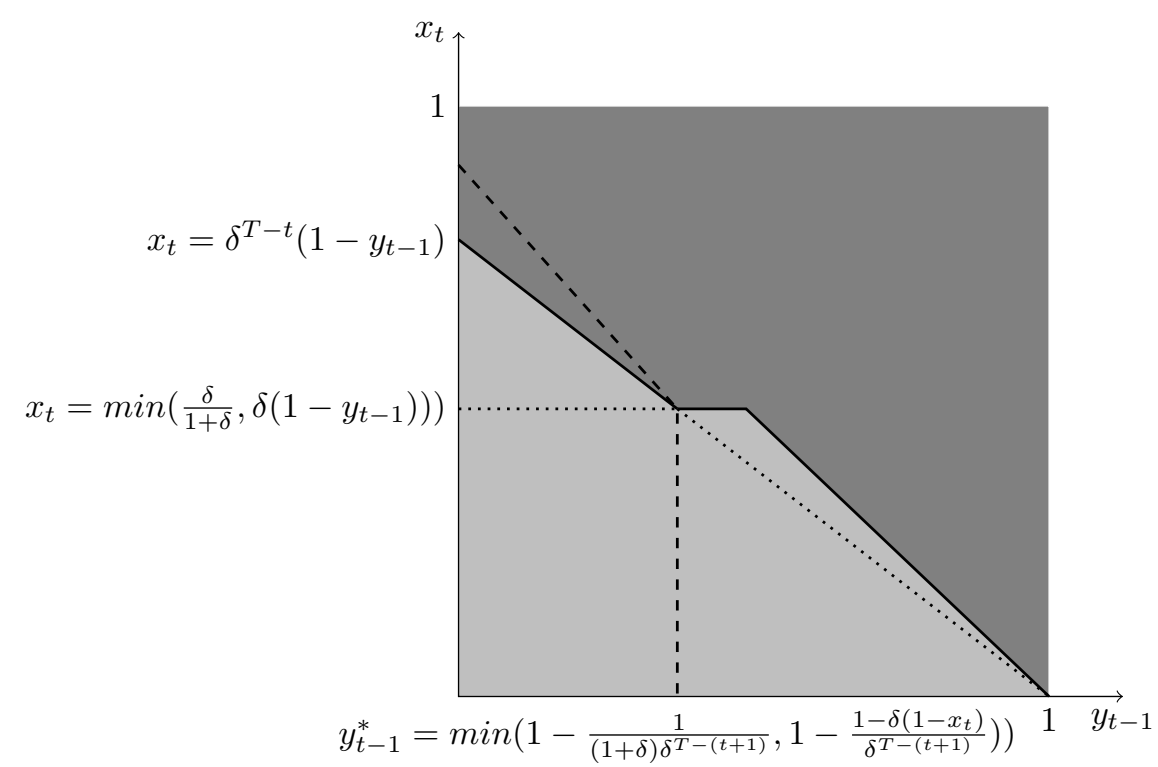

Figure 3: 2's response to $x_{t}$ given $y_{t-1}$

Figure 3 depicts cases (B) and (C). At the right-hand side of the dashed curve $\left(y_{t-1}>y_{t-1}^{*}\right)$, the minimal acceptable offer is $\left.x_{t}=\min \left(\frac{\delta}{1+\delta}, \delta\left(1-y_{t-1}\right)\right)\right)$. At the left-hand side of the dashed curve $\left(y_{t-1}<y_{t-1}^{*}\right)$, the minimal acceptable offer is $x_{t}=\delta^{T-t}\left(1-y_{t-1}\right)$. The dark gray area means that the offer will be accepted, and the light gray area means otherwise. The boundary between them is $x_{t}^{\# \#}$.

Combining cases (A) to (C), 2's best response is accepting if and only if $x_{t} \geq x_{t}^{\#}$ when $\delta \leq \delta_{t}^{*}$, and accepting if and only if $x_{t} \geq x_{t}^{\# \#}$ when $\delta>\delta_{t}^{*}$.

We next study 1 's best responses in $t$. Notice that if 1 makes an unacceptable offer in $t$, then 1 accepts 2's proposal in $t+1$ in cases (D) to (F), and 2 accepts 1 's proposal in $t+2$ in case $(\mathrm{G})$.

(D) When $\delta \leq \delta_{t}^{*}, 2$ accepts if and only if $x_{t} \geq x_{t}^{\#}$. When $x_{t-2} \geq x_{t}^{\#}, 1$ is bound to make acceptable offers, so he offers $x_{t}=x_{t-2}$. When $x_{t-2}<x_{t}^{\#}$, 1 earns $1-x_{t}$ if he offers $x_{t} \geq x_{t}^{\#}$ and $\operatorname{\delta max}\left(y_{t-1}, y_{t+1}^{\#}\right)$ if he offers $x_{t}<x_{t}^{\#}$. Therefore, 1 offers $x_{t}=x_{t}^{\#}$ if and only if

$$
1-x_{t}^{\#} \geq \delta \max \left(y_{t-1}, y_{t+1}^{\#}\right) \text {. }
$$


Notice that $1-x_{t}^{\#}=1-\min \left(\frac{\delta}{1+\delta}, \delta\left(1-y_{t-1}\right)\right)>\max \left(\frac{\delta}{1+\delta}, 1-\delta\left(1-y_{t-1}\right)\right) \geq$ $\max \left(\delta y_{t-1}, \frac{\delta}{1+\delta}\right) \geq \delta \max \left(y_{t-1}, \min \left(\frac{\delta}{1+\delta}, \delta\left(1-x_{t}\right)\right)\right)$; inequality 1 is valid. 1 offers $x_{t}=\max \left(x_{t-2}, x_{t}^{\#}\right)$.

(E) When $\delta>\delta_{t}^{*}$ and $y_{t-1}>\frac{\delta}{1+\delta}$, the analysis is similar to case (D), except that now inequality 1 becomes

$$
1-\delta\left(1-y_{t-1}\right) \geq \delta \max \left(y_{t-1}, y_{t+1}^{\#}\right)
$$

As $1-\delta\left(1-y_{t-1}\right) \geq \delta y_{t-1}$ and $1-\delta\left(1-y_{t-1}\right)>1-\left(1-y_{t-1}\right)=y_{t-1}>\frac{\delta}{1+\delta}>$ $\frac{\delta^{2}}{1+\delta} \geq \delta y_{t+1}^{\#}$, inequality 2 is valid. 1 offers $x_{t}=\max \left(x_{t-2}, \delta\left(1-y_{t-1}\right)\right)$.

(F) When $\delta>\delta_{t}^{*}$ and $1-\frac{1}{(1+\delta) \delta^{T-t-1}}<y_{t-1} \leq \frac{\delta}{1+\delta}$, the analysis is similar to case (D), except that now inequality 1 becomes

$$
1-\frac{\delta}{1+\delta} \geq \delta \max \left(y_{t-1}, y_{t+1}^{\#}\right)
$$

As $1-\frac{\delta}{1+\delta}=\frac{1}{1+\delta}>\frac{\delta^{2}}{1+\delta} \geq \delta y_{t-1}$ and $1-\frac{\delta}{1+\delta}=\frac{1}{1+\delta}>\frac{\delta^{2}}{1+\delta} \geq \delta y_{t+1}^{\#}$, inequality 3 is valid. 1 offers $x_{t}=\max \left(x_{t-2}, \frac{\delta}{1+\delta}\right)$.

(G) When $\delta>\delta_{t}^{*}$ and $y_{t-1} \leq 1-\frac{1}{(1+\delta) \delta^{T-t-1}}$, the analysis is similar to case (D), except that now inequality 1 becomes

$$
1-\delta^{T-t}\left(1-y_{t-1}\right) \geq \delta^{2}\left(1-\max \left(x_{t}, x_{t+2}^{\# \#}\right)\right) .
$$

$y_{t-1} \leq 1-\frac{1}{(1+\delta) \delta^{T-t-1}} \Rightarrow \delta^{T-t-2}\left(1-y_{t-1}\right)>\frac{1}{\delta(1+\delta)}>\frac{\delta}{1+\delta} \Rightarrow \max \left(\delta^{T-t-2}(1-\right.$ $\left.\left.y_{t-1}\right), \frac{\delta}{1+\delta}\right)=\delta^{T-t-2}\left(1-y_{t-1}\right)$, so the right-hand side of inequality 4 becomes $\delta^{2}\left(1-\max \left(x_{t}, \delta^{T-t-2}\left(1-y_{t-1}\right)\right)\right)$ (notice that 2 offers $y_{t+1} \geq y_{t-1}$ in $\left.t+1\right)$. As $1-\delta^{T-t}\left(1-y_{t-1}\right)>\delta^{2}-\delta^{T-t}\left(1-y_{t-1}\right)>\delta^{2}\left(1-\delta^{T-t-2}\left(1-y_{t-1}\right)\right)$, inequality 4 is valid. Therefore, 1 offers $x_{t}=\max \left(x_{t-2}, \delta^{T-t}\left(1-y_{t-1}\right)\right)$.

Combining cases (D) to $(\mathrm{G}), 1$ 's best response is offering $x_{t}=\max \left(x_{t-2}, x_{t}^{\#}\right)$ when $\delta \leq \delta_{t}^{*}$, and offering $x_{t}=\max \left(x_{t-2}, x_{t}^{\# \#}\right)$ when $\delta>\delta_{t}^{*}$. Both players' best responses coincide with $(f, g)$.

Now suppose that 2 proposes in $t$. In $t^{\prime}>t$, when $\delta \leq \delta_{t+1}^{*}, 2$ accepts $x_{t+1}=$ $x_{t+1}^{\#}$ in $t+1$ and the bargaining outcome is $\left.\left(1-\max \left(x_{t-1}, x_{t+1}^{\#}\right)\right), \max \left(x_{t-1}, x_{t+1}^{\#}\right)\right)$. When $\delta>\delta_{t+1}^{*}, 2$ accepts $x_{t+1}=x_{t+1}^{\# \#}$ in $t+1$ and the bargaining outcome is $\left(1-\max \left(x_{t-1}, x_{t+1}^{\# \#}\right), \max \left(x_{t-1}, x_{t+1}^{\# \#}\right)\right)$. We first study 1 's best responses in $t$.

(H) When $\delta \leq \delta_{t+1}^{*}, 1$ accepts if and only if $y_{t} \geq \delta\left(1-\max \left(x_{t-1}, x_{t+1}^{\#}\right)\right)$, namely, $y_{t} \geq y_{t}^{\#}=\min \left(\frac{\delta}{1+\delta}, \delta\left(1-x_{t-1}\right)\right)$.

(I) When $\delta>\delta_{t+1}^{*}, 1$ accepts if and only if $y_{t} \geq \delta\left(1-\max \left(x_{t-1}, x_{t+1}^{\# \#}\right)\right)$, namely, $y_{t} \geq y_{t}^{\#}=\min \left(\frac{\delta}{1+\delta}, \delta\left(1-x_{t-1}\right)\right)$.

Combining cases (H) and (I), 1's best response is accepting if and only if $y_{t} \geq y_{t}^{\#}$.

We next study 2's best responses in $t$. 
(J) When $\delta \leq \delta_{t+1}^{*}$, the analysis is similar to case (D), except that now inequality 1 becomes

$$
1-y_{t}^{\#} \geq \delta \max \left(x_{t-1}, x_{t+1}^{\#}\right)
$$

which is equivalent to inequality 1 . It's easy to derive that 2 offers $y_{t}=$ $\max \left(y_{t-2}, y_{t}^{\#}\right)$.

(K) When $\delta>\delta_{t+1}^{*}$, the analysis is similar to case (D), except that now inequality 1 becomes

$$
1-y_{t}^{\#} \geq \delta \max \left(x_{t-1}, x_{t+1}^{\# \#}\right) .
$$

Take a look at $x_{t+1}^{\# \#}=\min \left(\max \left(\delta^{T-t-1}\left(1-y_{t}\right), \frac{\delta}{1+\delta}\right), \delta\left(1-y_{t}\right)\right)$. Obviously, the highest payoff reaches when $y_{t}=y_{t-2}$. When $\delta^{T-t-1}\left(1-y_{t-2}\right) \leq \frac{\delta}{1+\delta}$, namely, $y_{t-2} \geq 1-\frac{1}{(1+\delta) \delta^{T-t-2}}$, inequality 6 becomes inequality 5 and is therefore valid. When $\delta^{T-t-1}\left(1-y_{t-2}\right)>\frac{\delta}{1+\delta}$, namely, $y_{t-2}<1-\frac{1}{(1+\delta) \delta^{T-t-2}}$, inequality 6 becomes $1-y_{t}^{\#} \geq \delta \max \left(x_{t-1}, \delta^{T-t-1}\left(1-y_{t-2}\right)\right)$. As $1-\min \left(\frac{\delta}{1+\delta}, \delta\left(1-x_{t-1}\right)\right) \geq$ $1-\delta\left(1-x_{t-1}\right)>\delta x_{t-1}$, it further becomes $1-y_{t}^{\#} \geq \delta^{T-t}\left(1-y_{t-2}\right)$, namely, $y_{t-2} \geq y_{t-2}^{*}=\min \left(1-\frac{1}{(1+\delta) \delta^{T-t}}, 1-\frac{1-\delta\left(1-x_{t-1}\right)}{\delta^{T-t}}\right)$. Notice that (a) when $\delta \leq \delta_{t}^{*}$, $1-\frac{1}{(1+\delta) \delta^{T-t}} \leq 0$, so inequality 6 must be valid; (b) when $\delta>\delta_{t}^{*}$ and $y_{t-2}<y_{t-2}^{*}$, $x_{t-1}<1-\frac{1-\delta^{T-t}\left(1-y_{t-2}\right)}{\delta}<\delta^{T-t-1}\left(1-y_{t-2}\right)$, so 2's payoff when making an unacceptable offer becomes $\delta^{T-t}\left(1-y_{t-2}\right)$; and (c) when 2 proposes, $T-t$ is even and $\delta_{t}^{*}>\delta_{t+1}^{*}$. Therefore, 2 offers $y_{t}=\max \left(y_{t-2}, y_{t}^{\#}\right)$ when $\delta_{t+1}^{*}<\delta \leq \delta_{t}^{*}$ or when $\delta>\delta_{t}^{*}$ and $y_{t-2} \geq y_{t-2}^{*}$, offers $y_{t}=y_{t-2}$ when $\delta>\delta_{t}^{*}$ and $y_{t-2}<y_{t-2}^{*}$.

Combining cases $(\mathrm{J})$ and $(\mathrm{K}), 2$ 's best response is offering $y_{t}=\max \left(y_{t-2}, y_{t}^{\#}\right)$ when $\delta \leq \delta_{t}^{*}$ or when $\delta>\delta_{t}^{*}$ and $y_{t-2} \geq y_{t-2}^{*}$, and offering $y_{t}=y_{t-2}$ when $\delta>\delta_{t}^{*}$ and $y_{t-2}<y_{t-2}^{*}$.

Clearly, both players' best responses coincide with $(f, g)$.

Now that $(f, g)$ specifies the best responses when $t=T, T-1, T-2$, and are the best responses in $t$ if they are the best responses in $t^{\prime}>t,(f, g)$ constitutes the unique sub-game perfect equilibrium.

Now we discuss the equilibrium without the tie-breaking rule.

For the respondent's indifference choices: Suppose the respondent rejects with a positive probability when indifferent. It is easy to imagine that the proposer has an incentive to increase the proposal by $\epsilon>0, \epsilon \rightarrow 0$ as the total payoffs strictly decrease with time.

For the proposer's indifference choices: The only time this happens is in case $(\mathrm{K})$, when $\delta_{t}>\delta_{t}^{*}$ and $y_{t-2}=y_{t}^{*}$. However, from our analysis of cases (B) and (C), we see that 2's indifference as proposer is resolved and does not affect her reservation value in the earlier period, where 1 makes an acceptable proposal $x_{t-1}=x_{t-1}^{\# \#}$ anyway. Thus such indifference cannot affect on-path play unless it occurs at $t=1$, when $T$ is odd and $\delta=\delta_{1}^{*}$. 


\section{References}

[1] Admati, A.R., Perry, M., 1991. Joint Projects without Commitment. Rev. Econ. Stud. 58, 259-276.

[2] Compte, O., Jehiel, P., 2003. Bargaining with Reference Dependent Preferences. Unpublished paper.

[3] Driesen, B., Perea, A., Peters, H., 2012. Alternating Offers Bargaining with Loss Aversion. Math. Soc. Sci. 64, 103-118.

[4] Fershtman, C., Seidmann, D., 1993. Deadline Effects and Inefficient Delay in Bargaining with Endogenous Commitment. J. Econ. Theory 321, 306-321.

[5] Li, D., 2007. Bargaining with History-Dependent Preferences. J. Econ. Theory $136,695-708$.

[6] Rubinstein, A., 1982. Perfect Equilibrium in a Bargaining Model. Econometrica 50, 97-109.

[7] Schwartz, J.A., Wen, Q., 2007. Wage Negotiation Under Good Faith Bargaining. Int. Game Theory Rev. 9, 551-564.

[8] Stahl, I. 1972. Bargaining Theory. Economics Research Institute, Stockholm School of Economics, Stockholm. 